\title{
Fuzzy Rule Based Approach with Z-Numbers for Selection of Alternatives using TOPSIS
}

\author{
Abdul Malek Yaakob \\ School of Quantitative Science \\ Universiti Utara Malaysia \\ 06010 Sintok Kedah Darul Aman, Malaysia \\ abd.malek@uum.edu.my
}

\author{
Alexander Gegov \\ School of Computing \\ University of Portsmouth \\ Buckingham Building, PO1 3HE, United Kingdom \\ alexander.gegov@port.ac.uk
}

\begin{abstract}
The lack of ability to handle vagueness in the decision making practice has been main drawback of the conventional TOPSIS. Thus, type 1 , type 2 and $Z$ fuzzy sets have been applied with conventional TOPSIS to allow experts to incorporate imperfect information in analysis. However the existing methods do not take into account the influence degree of decision makers. Hence, a novel modification of TOPSIS method to handle vagueness and imperfect information in decision making practice is presented. The concept of $Z$ - numbers is used to present decision maker's reliability. Furthermore, a hybrid analysis of decision making process that requires the use of human sensitivity to reflect influence degree of decision maker can be often expressed by a fuzzy rule base. The ranking based on proposed method is validated comparatively using Spearmen rho correlation coefficient. The result shows proposed method outperforms the existing non rule based version of TOPSIS in terms of ranking performance.
\end{abstract}

Keywords-TOPSIS; decision making; ranking alternative; fuzzy rule based system; Z-Numbers; decision maker's reliability; influence degree; influence multiplier, ranking performance

\section{INTRODUCTION}

There has been an increasing interest in group decision making technique and a considerable amount of study has been published on it. In about forty years since it is introduced , over 70 Multi Criteria Decision Making (MCDM) techniques has developed for facilitating decision making practice [1]. TOPSIS which was introduced in 1981 is a helpful technique in dealing with MCDM problems in real life. It helps Decision Makers (DMs) solve the problem through analysis, comparisons and rankings of the alternatives. TOPSIS has been deemed one of the major decision making techniques. In recent years, TOPSIS has been effectively applied to the areas of human resources management [2], transportation [3], product design [4], manufacturing [5], water management [6], quality control [7], military [8], tourism [9] and location analysis [10].

According to [11]and [12], TOPSIS has the following three advantages: (i) a sound logic that represents the rationale of individual choice; (ii) a scalar value that record for both the best and worst alternatives concurrently; and (iii) a straight forward computation algorithm that can be easily programmed into a spreadsheet. In fact, TOPSIS is a value-based process that compares each alternative directly depending on information in the evaluation matrices and weights [5].Thus, TOPSIS is chosen as the main body of development in this study. However, the main drawback of conventional TOPSIS lies in the lack of ability to handle vagueness and imperfect information in decision making practice. In order to overcome this shortcoming, the fuzzy sets i.e. type 1; type 2 and Z-fuzzy set were applied with conventional TOPSIS. In 2000 and 2010, type-1 TOPSIS, type-2 TOPSIS and Z-TOPSIS were proposed in [13], [14] and [15] respectively. Nevertheless, the reliability of the decision information and the experience of the expert are not well taken into consideration in decision process. Therefore the problems arise how sure the decision makers are about their decision and how much experience the experts have in the field. These are the motivation of this study.

In this paper, the concept of Z-numbers introduced in [16] is used which can deal with the reliability of decision. In addition to that, the presentation of influence degree representing the level experience of the expert by fuzzy rule based approach. It seems to be more flexible and intuitively significant for formalizing information structure of a decision making practice. In section II, theoretical preliminaries for fuzzy sets are reviewed. The proposed method is explained systematically in Section III, with various combinations in a step-by-step fashion. Afterwards, the case study on stock selection problem is conducted to illustrate the usefulness of proposed method in section IV. Then, in section $\mathrm{V}$ the ranking are assessed descriptively using spearman's rank correlation. In the final section, conclusions are drawn.

\section{BASIC CONCEPT}

In the following, some basic definitions of fuzzy sets from [13]-[15] is briefly review. These basic definitions and 
notations are used throughout the paper unless stated otherwise.

\section{Definition 1: Fuzzy set}

A fuzzy set $A$ is defined on a universe $X$ may be given as:

$$
A=\left\{\left(x, \mu_{A_{i}}(x)\right) \mid x \in X\right\}
$$

Where $\mu_{A_{i}}(x): X \rightarrow[0,1]$ is the membership function of $A$. The membership value $\mu_{A_{i}}(x)$ describes the degree of belongingness of $x \in X$ in $A$. Throughout this paper, type-1 fuzzy number and Z-number are presented in the form of trapezoidal fuzzy number. It is easy to deal with because it is piece wise linear. On the other hand, the good coverage of trapezoidal fuzzy number is a good compromise between efficiency and effectiveness.

\section{Definition 2: Type-1 Fuzzy Number}

A trapezoidal fuzzy numbers can be represented by the following membership function given by

$$
\mu_{A_{i}}(x)=\left(a_{i 1}, a_{i 2}, a_{i 3}, a_{i 4}\right)=\left\{\begin{array}{cll}
\frac{x-a_{i 1}}{a_{i 2}-a_{i 1}} & \text { if } & a_{i 1} \leq x \leq a_{i 2} \\
1 & \text { if } & a_{i 2} \leq x \leq a_{i 3} \\
\frac{a_{i 4}-x}{a_{i 4}-a_{i 3}} & \text { if } & a_{i 3} \leq x \leq a_{i 4} \\
0 & & \text { otherwise }
\end{array}\right.
$$

Fig.1. Type-1 Fuzzy Number

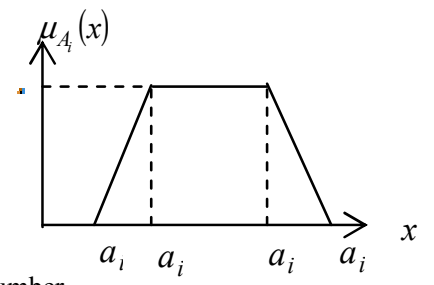

\section{Definition 2: Z-numbers}

Z-number is an ordered pair of type-1 fuzzy numbers denoted as $Z=(\widetilde{A}, \widetilde{B})$. The first component $\widetilde{A}$, a restriction on the values, is a real-valued uncertain variable. The second component $\widetilde{B}$ is a measure of reliability for the first component. The illustration of the $Z-$ number can be described as Figure 3.

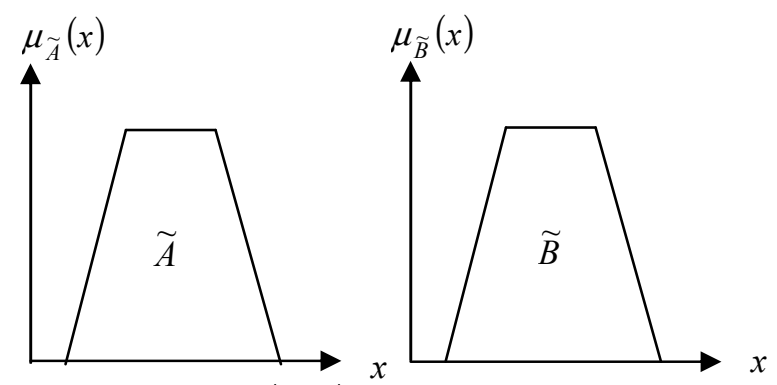

Fig.2. $Z$ - Number, $Z=(\widetilde{A}, \widetilde{B})$

The concept of a Z-numbers $Z=(\widetilde{A}, \widetilde{B})$ is intended to provide a basis for computation with numbers which not totally reliable. A Z-number can be used to represent the information about an uncertain variable of the type where $\tilde{A}$ represents a value of the variable $X$, and the second component, $\widetilde{B}$ represent an idea of certainty or probability such as the concept of sureness, confident, reliability, strength of belief and possibilities. Or informally, B may be interpreted as a response to the question: How sure are decision makers that $\mathrm{X}$ is $\mathrm{A}$. Example of $\mathrm{Z}$-valuation are:

(Very good, Likely), (Good, Unlikely)

\section{PROPOSED METHOD}

In this section, the authors have modified the Z-TOPSIS method introduced in [15] using fuzzy rule based approach. Basically, the concepts of Z-Numbers have capability to represent the reliability of decision maker into decision making evaluation. In order to enhance capability to deal with vagueness and representing decision information more effectively, in this paper the authors propose additional element that represent the evaluation of a fuzzy rule based systems in Z-TOPSIS. The main objective of this modification is to introduce the ability of fuzzy rule based system in existing Z-TOPSIS. Thus, the evaluation by proposed method allows the empirical knowledge of the expert, represented by fuzzy rule, also can be take in to account in the decision making process.

The use of technique associated with the empirical knowledge of experts, allows us a hybrid analysis of the decision making problems where the process of decision making requires the use of human sensitivity, which often can be expressed by a fuzzy rules base. The influence level of this rule is defined by influence degree that the criteria will receive in the analysis of the problem. The authors have adopt the methods described in [17] for the knowledge of the influence degree of each decision maker. In case in which one decision maker has more knowledge of the domain, optionally the opinion of this expert may have greater degree of importance than the other decision makers in the analysis of the problem. Thus, the proposed method can identify and aggregate the different opinions of decision makers with varying influence degrees to suggest the final solution.

As established fuzzy TOPSIS method [13], Table 1 and Table 2 are used in representing the importance of criteria and the rating of the alternative. In addition to that, Table 3 and Table 4 are proposed in this paper is used to represent the reliability of decision makers and to identify the alternative level for the consequent part of the rule.

TABLE 1: LINGUISTIC VARIABLE FOR IMPORTANCE WEIGHT OF EACH CRITERION

\begin{tabular}{|l|c|}
\hline \multicolumn{1}{|c|}{ Linguistic Variables } & Trapezoidal Fuzzy Number \\
\hline Very Low (VL) & $(0.00,0.00,0.00,0.10)$ \\
\hline Low (L) & $(0.00,0.10,0.10,0.25)$ \\
\hline Medium Low (ML) & $(0.15,0.30,0.30,0.45)$ \\
\hline Medium (M) & $(0.35,0.50,0.50,0.65)$ \\
\hline Medium High (MH) & $(0.55,0.70,0.70,0.85)$ \\
\hline High (H) & $(0.80,0.90,0.90,1.00)$ \\
\hline Very High (VH) & $(0.90,1.00,1.00,1.00)$ \\
\hline
\end{tabular}


TABLE 2: LINGUISTIC VARIABLE FOR RATING OF ALL ALTERNATIVE

\begin{tabular}{|l|c|}
\hline \multicolumn{1}{|c|}{ Linguistic Variables } & Trapezoidal Fuzzy Number \\
\hline Very Poor (VP) & $(0,0,0,1)$ \\
\hline Poor (P) & $(0,1,1,3)$ \\
\hline Medium Poor (MP) & $(1,3,3,5)$ \\
\hline Fair (F) & $(3,5,5,7)$ \\
\hline Medium Good (MG) & $(5,7,7,9)$ \\
\hline Good (G) & $(7,9,9,10)$ \\
\hline Very Good (VG) & $(9,10,10,10)$ \\
\hline
\end{tabular}

TABLE 3: LINGUISTIC VARIABLE FOR EXPERT'S RELIABILITY

\begin{tabular}{|l|c|}
\hline \multicolumn{1}{|c|}{ Linguistic Variables } & Trapezoidal Fuzzy Number \\
\hline Strongly Unlikely (SU) & $(0.00,0.00,0.00,0.10)$ \\
\hline Unlikely (U) & $(0.00,0.10,0.10,0.25)$ \\
\hline Somewhat Unlikely (SWU) & $(0.15,0.30,0.30,0.45)$ \\
\hline Neutral (N) & $(0.35,0.50,0.50,0.65)$ \\
\hline Somewhat Likely (SWL) & $(0.55,0.70,0.70,0.85)$ \\
\hline Likely (L) & $(0.80,0.90,0.90,1.00)$ \\
\hline Strongly Likely (SL) & $(0.90,1.00,1.00,1.00)$ \\
\hline
\end{tabular}

The linguistic variable that represents the consequents of rules was named "Alternative Level" and is represented by fuzzy sets "Very bad", "Bad", "Regular", "Good" and "Excellent".

TABLE 4: LINGUISTIC VARIABLE FOR ALTERNATIVE LEVEL

\begin{tabular}{|l|c|}
\hline \multicolumn{1}{|c|}{ Linguistic Variables } & Trapezoidal Fuzzy Number \\
\hline Very Bad (VB) & $(0.00,0.00,0.00,0.25)$ \\
\hline Bad (B) & $(0.00,0.25,0.25,0.50)$ \\
\hline Regular (R) & $(0.25,0.50,0.50,0.75)$ \\
\hline Good (G) & $(0.50,0.75,0.75,1.00)$ \\
\hline Very Good (VG) & $(0.75,1.00,1.00,1.00)$ \\
\hline
\end{tabular}

The following algorithm is conducted to obtain the ranking of alternatives, whereby Step 1 is purely from [18] but it make use the linguistics variable for expert's reliability from Table 3 for the component B in Z-Numbers, follows by Step 2- 7 are adopted from [13]. In order to deal with influence degree of decision maker the authors have introduced the step 8-10, which make use the rule based approach.

\section{Z- FRBS TOPSIS Algorithm}

Instead of calculating the average decision matrix as previous TOPSIS methods in [2],[13]. In here, opinion of each decision maker evaluated independently. Assume there are $m$ alternatives $A_{1}, A_{2}, \cdots, A_{m}$ and there are $n$ criteria $C_{1}, C_{2}, \cdots, C_{n}, C_{n+1}$. Where $C_{n+1}$ represent the influence level of each decision maker. Let $k$ decision makers $D M_{1}, D M_{2}, \cdots, D M_{k}$ then will have $k$ decision matrix.

Step 1: Used the information from Table 3 to derive component $\mathrm{B}$, and then convert Z-numbers to type-1 fuzzy number

Assume a Z-number, $Z=(\widetilde{A}, \widetilde{B})$.Let

$\left\{\widetilde{A}=\left(x, \mu_{\widetilde{A}}\right) \mid x \in[0,1]\right\},\left\{\widetilde{B}=\left(x, \mu_{\widetilde{B}}\right) \mid x \in[0,1]\right\}, \mu_{\widetilde{A}}$ and

$\mu_{\widetilde{B}}$ is a trapezoidal membership function. The second part (reliability) needs to convert into crisp number using fuzzy expectation as

$$
\alpha=\frac{\int x \mu_{\widetilde{B}} d x}{\int \mu_{\widetilde{B}} d x}
$$

where $\int$ denotes an algebraic integration. Then add the weight of the second part (reliability) to the first part (restriction). Weighted Z-number can be denoted as

$$
\widetilde{Z}^{\alpha}=\left\{\left(x, \mu_{\widetilde{A}^{\alpha}}\right) \mid \mu_{\widetilde{A}^{\alpha}}(x)=\alpha \mu_{\widetilde{A}}(x), x \in[0,1]\right\}
$$

These can be type-1 fuzzy number as

$$
\widetilde{Z}^{\prime}=\left\{<x, \mu_{\widetilde{Z}^{\alpha}}(x)>\mid \mu_{\widetilde{Z}^{\alpha}}(x)=\mu_{\widetilde{A}}\left(\frac{x}{\sqrt{\alpha}}\right), x \in[0,1]\right\}
$$

It is proven in [19] that $\widetilde{Z}^{\prime}$ has the same Fuzzy Expectation with $\widetilde{Z}^{\alpha}$.

Step 2: Construct decision matrix $\widetilde{D}$ and weight matrix $\widetilde{W}$

Assume that a decision group has $\mathrm{K}$ persons, and then the importance of the criteria and the rating of alternatives with respect to each criterion. Multi criteria decision making problem can easily expressed in matrix format as

$$
\begin{aligned}
& \widetilde{D}=\left[\begin{array}{cccc}
\tilde{x}_{11} & \tilde{x}_{12} & \cdots & \tilde{x}_{1 n} \\
\tilde{x}_{21} & \tilde{x}_{22} & \cdots & \tilde{x}_{2 n} \\
\vdots & \vdots & \ddots & \vdots \\
\tilde{x}_{m 1} & \tilde{x}_{m 2} & \cdots & \tilde{x}_{m n}
\end{array}\right] \\
& \tilde{W}=\left[\begin{array}{llll}
\widetilde{w}_{1} & \widetilde{w}_{2} & \cdots & \widetilde{w}_{n}
\end{array}\right]
\end{aligned}
$$

Where $\tilde{x}_{i j}$ for all $i=(1,2, \ldots n), j$ and $\widetilde{w}_{j}$ are linguistic variables. These linguistic variables can be described by fuzzy numbers, $\tilde{x}_{i j}=\left(a_{i j}, b_{i j}, c_{i j}, d_{i j}\right)$ and $\widetilde{w}_{j}=\left(w_{j 1}, w_{j 2}, w_{j 3}, w_{j 4}\right)$.

Step 3: Construct normalized fuzzy decision matrix, $\widetilde{R}$ and normalized decision matrix $\tilde{V}$

For the purpose of making various scales comparable, linear scale transformation is used to construct normalized fuzzy decision matrix as

$$
\widetilde{R}=\left[\widetilde{r}_{i j}\right]_{m \times n}
$$

where $\mathrm{B}$ and $\mathrm{C}$ are the set of benefit criteria and cost criteria, respectively, and

$$
\begin{gathered}
\widetilde{r}_{i j}=\left(\frac{a_{j}^{-}}{d_{i j}}, \frac{a_{j}^{-}}{c_{i j}}, \frac{a_{j}^{-}}{b_{i j}}, \frac{a_{j}^{-}}{a_{i j}},\right), j \in B ; \\
\tilde{r}_{i j}=\left(\frac{a_{i j}}{c_{j}^{*}}, \frac{b_{i j}}{c_{j}^{*}}, \frac{c_{i j}}{c_{j}^{*}}, \frac{d_{i j}}{c_{j}^{*}}\right) \quad j \in C
\end{gathered}
$$




$$
\begin{aligned}
& d_{j}^{*}=\max _{i} d_{i j} \text { if } j \in B ; \\
& a_{j}^{-}=\min _{i} a_{i j} \text { if } j \in C ;
\end{aligned}
$$

The technique mentioned on top of is to preserve the property that the ranges of normalized fuzzy numbers belong to $[0,1]$.

Considering the different importance of each criterion, we can construct the weighted normalized fuzzy decision matrix as

$$
\begin{gathered}
\widetilde{V}=\left[\widetilde{v}_{i j}\right]_{m \times n} i=1,2, \ldots, m \text { and } j=1,2, \ldots, n \\
\text { where } \widetilde{v}_{i j}=\widetilde{r}_{i j}(\cdot) \widetilde{w}_{j} .
\end{gathered}
$$

Step 4: Find Fuzzy Positive-Ideal Solution, $A^{*}$ and Fuzzy Negative-Ideal Solution, $A^{-}$

Based on the weighted normalized fuzzy decision matrix, the elements $\widetilde{v}_{i j}$, for all $i$ and $j$ are normalized positive triangular fuzzy numbers and their ranges belong to the closed interval $[0,1]$. Then, we can define the fuzzy positive-ideal solution and fuzzy negative-ideal solution as

$$
\begin{aligned}
& A^{*}=\left(\widetilde{v}_{1}^{*}, \widetilde{v}_{2}^{*}, \ldots, \tilde{v}_{n}^{*}\right), \\
& A^{-}=\left(\widetilde{v}_{1}^{-}, \widetilde{v}_{2}^{-}, \ldots, \widetilde{v}_{n}^{-}\right),
\end{aligned}
$$

where $\widetilde{v}_{j}^{*}=(1,1,1,1)$ and, $\widetilde{v}_{j}^{-}=(0,0,0,0)$ for $j=1,2, \ldots, n$.

Find Distance of Each Alternative from $A^{*}$ and $A^{-}$

The distance of each alternative from $A^{*}$ and $A^{-}$can be currently calculated as

$$
\begin{aligned}
& d_{i}^{*}=\sum_{j=1}^{n} d\left(\tilde{v}_{i j}, \tilde{v}_{j}^{*}\right), i=1,2, \ldots, m, \\
& d_{i}^{-}=\sum_{j=1}^{n} d\left(\tilde{v}_{i j}, \tilde{v}_{j}^{-}\right), i=1,2, \ldots, m,
\end{aligned}
$$

where $d(\cdot, \cdot)$ is the distance measurement between two fuzzy numbers.

\section{Step 5: Find Closeness Coefficient, $C C_{i}$}

A closeness coefficient is defined to determine the ranking order of all alternatives once the $d_{i}^{*}$ and $d_{i}^{-}$of each alternative $A_{i}$ for $i=1,2, \ldots, m$ has been calculated. The closeness coefficient of each alternative is calculated as

$$
C C_{i}=\frac{d_{i}^{-}}{d_{i}^{*}+d_{i}^{-}}, i=1,2, \ldots, m
$$

Step 6: The Influence Closeness Coefficient $I C C_{i}$ of each alternative
The influence degree of each decision maker has been defined at this point, noting that experts with more experience may have a greater degree of influence than the others.

$$
\text { Let } \sigma_{K}=\theta_{i} / \sum_{i=1}^{K} \theta_{i} \text { for } i=1, \cdots, m
$$

Where $\sigma_{K}$ represent normalized influence degree for $K^{\text {th }}$ decision maker. $\theta_{i}$ is the importance degree between 0( unimportant) and 10 (very importance) of decision maker. Then

$$
I C C_{i}=\sigma_{K} * C C_{i}
$$

And it is necessary to normalized the $I C C_{i}\left(N I C C_{i}\right)$ to ensure that the $I C C_{i}$ value varies between 0 to 1 .

$$
N I C C_{i}=I C C_{i} / \max _{i} I C C_{i}
$$

Step 7: The matrix of antecedent $(\Lambda)$ and the matrix of consequent $(\chi)$

To determine a matrix with antecedents is defined as

$$
\Lambda=\left[\begin{array}{cccc}
X_{11} & X_{12} & \cdots & X_{1 n} \\
X_{21} & X_{22} & \cdots & X_{2 n} \\
\vdots & \vdots & \ddots & \vdots \\
X_{m 1} & X_{m 2} & \cdots & X_{m n}
\end{array}\right]
$$

where $X_{i j}$ is a linguistic variable represent the decision maker opinion of each alternative respect to the criteria.

Once $N I C C_{i}$ for each alternative defined by each decision maker is obtained, it is used to determine the consequents of alternative rules according to the fuzzy set with higher membership in Table 4. Then a matrix of consequents is define as follow

$$
\chi=\left[\begin{array}{c}
Y_{1} \\
Y_{2} \\
\vdots \\
Y_{m}
\end{array}\right]
$$

where $Y_{j}$ is a linguistic variable based on Table 4 representing the output of the system based on Eq. (10) to find the value of $\mathrm{NICC}_{i}$.

Step 8: The final score $(\Gamma)$ for each alternative

$$
\Gamma=\lambda * \Omega
$$

Where $\lambda$ is a crisp value of aggregate membership function of the output defined as

$$
\lambda=\sum_{i=1}^{K} \alpha_{i j} / K
$$


Where the value of $\alpha_{i j} \in Y_{j}$ is maximum membership degree of the output. In order to obtained a better representation in the ranking made by Z- FRBS TOPSIS. It is importance to have influence multiplier when the alternatives have same ranking position. It is show exactly how each alternative is different even a small difference. The following general formula to calculate influence multiplier $(\Omega)$ uses marginal closeness coefficient that have maximum membership degree.

$$
\Omega=\sum_{i=1}^{K} N I C C_{i} / K
$$

Therefore, from the value of $\Gamma$, the ranking order of all alternative can be determine. The best alternative has higher value of $\Gamma$.

\section{CASE STUDY}

In this case study a stock selection problem is considered in which the evaluation has been done by three decision makers. These financial experts including finance lecturer (DM1), fund manager (DM2) and PhD finance student (DM3). They evaluated 25 stocks listed on Main Board in Kuala Lumpur Stock Exchange at 30 November 2007 and then made investment recommendations according to financial ratio considered.

Microsoft Excel is used to calculate all the calculation involved to evaluate ranking of stocks and weight of each criterion. The DMs uses the linguistic weighting variable in Table 1 to assess the importance of the criteria, make use information in Table 2 to give rating for each alternative. Then use the information from Table 3 to express their reliability. The Z- FRBS TOPSIS algorithm introduced in Section 3 is now illustrated for the stock selection analysis.

Step 1: Used the information from Table 3 to derive component B, and then convert Z-number to type-1 fuzzy number.

In this step, $\mathrm{C} 1$ from Table 5 is used to illustrate the procedure of our approach. Assume Decision Maker 1 (DM1) give his opinion as follows:

$$
\begin{aligned}
\widetilde{A} & =(0.9,1.0,1.0,1.0 ; 1) \\
\widetilde{B} & =(0.8,0.9,0.9,1.0 ; 1)
\end{aligned}
$$

The DMs knowledge can be expressed to Z-number as:

$$
\widetilde{Z}=[(0.9,1.0,1.0,1.0 ; 1),(0.8,0.9,0.9,1.0 ; 1)]
$$

At first, we should convert DMs reliability into crisp number

$$
\alpha=\frac{\int x \mu_{\widetilde{B}} d x}{\int \mu_{\widetilde{B}} d x}=0.9
$$

Second, add the weight of reliability to the constraint.

$$
\widetilde{Z}^{\alpha}=(0.9,1 \cdot 0,1.0,1.0 ; 0.9)
$$

Third, convert the weighted Z-number to type-1 fuzzy number according to proposed approach.

$$
\begin{aligned}
\widetilde{Z}^{\prime} & =(\sqrt{0.9} * 0.9,(\sqrt{0.9} * 1.0,(\sqrt{0.9} * 1.0,(\sqrt{0.9} * 1.0) \\
& =(0.81,0.9,0.9,0.9)
\end{aligned}
$$

Repeat the same procedure for all DM's judgments.

Step 2: Construct Z- decision matrix, $(\widetilde{D})$ and Z-weight of alternative. $(\tilde{W})$

Converting the linguistic evaluation shown in table 5 and table 6 in the form of trapezoidal fuzzy number to construct the fuzzy decision matrix and determine the fuzzy weight of each criterion. The important of criteria and the rating of each alternative presented in Table 5, Table 6 (A), Table 6 (B) and Table $6(\mathrm{C})$ are obtained from questionnaire.

Step 3: Construct a normalized Z-decision matrix $(\widetilde{R})$ and weight normalize Z-decision making matrix $(\widetilde{V})$

In order to construct fuzzy weighted normalized fuzzy decision matrix, Eq. (4) is used.

$$
\begin{array}{ll}
\tilde{v}_{i j}=(a, b, c, d) & \\
a=4.50 * 0.81 & b=5.0 * 0.90 \\
a=3.65 & b=4.5 \\
c=5.0 * 0.90 & d=5.0 * 0.90 \\
c=4.5 & d=4.5
\end{array}
$$

Therefore the $\widetilde{v}_{11}=(3.65,4.50,4.50,4.50)$

From the decision matrix obtained in previous step, the $\max _{i} C_{1 j}=9.35$, and using Eq. (3) stated to construct the normalized fuzzy decision matrix.

$$
\begin{aligned}
& \widetilde{r}_{11}=\left(\frac{3.65}{9.35}, \frac{4.50}{9.35}, \frac{4.50}{9.35}, \frac{4.50}{9.35}\right) \\
& \widetilde{r}_{11}=(0.3900,0.4815,0.4815,0.4815,0.4815)
\end{aligned}
$$

The normalization method mentioned above is to preserve the property, the range of normalized trapezoidal fuzzy number belong to $[0,1]$.

Step 4: The fuzzy positive-ideal solution $A^{*}$ and fuzzy negative-ideal solution $A^{-}$

Determine FPIS and FNIS as

$$
\begin{aligned}
& A^{*}=\left[(1,1,1,1)_{1},(1,1,1,1)_{2}, \ldots,(1,1,1,1,)_{25}\right], \\
& A^{-}=\left[(0,0,0,0)_{1},(0,0,0,0)_{2}, \ldots,(0,0,0,0)_{25}\right] .
\end{aligned}
$$

Distance of each alternative from $\widetilde{A}^{*}$ and $\widetilde{A}^{-}$

The distance of alternative from FPIS and FNIS are shown below.

Using Eq. (6) fuzzy positive ideal solution for S1 calculated as follows

$d\left(C_{11}, A^{+}\right)=\sqrt{\frac{1}{3}\left[(0.39-1)^{2}+\cdots+(0.4815-1)^{2}\right]}=0.7563$

Hence, similarly 
$d\left(C_{12}, A^{+}\right)=1.0241$

$d\left(C_{13}, A^{+}\right)=0.9114 d\left(C_{14}, A^{+}\right)=0.7629$,

$d\left(C_{15}, A^{+}\right)=0.3485$ and $d\left(C_{16}, A+\right)=1.213$

$D_{11}^{+}=0.7563+1.0241+0.9114+0.762+0.3485+1.213=5.015$

Next, using Eq. (6) Fuzzy negative ideal solution for

S1calculated as

$$
d\left(C_{11}, A^{-}\right)=\sqrt{\frac{1}{3}\left[(0.39-0)^{2}+\cdots+(0.4815-0)^{2}\right]}=0.6612
$$

Repeat the same formula for the following

$d\left(C_{12}, A^{-}\right)=0.3983, d\left(C_{12}, A^{-}\right)=0.3983$,

$d\left(C_{13}, A^{-}=0.5266, d\left(C_{14}, A^{-}\right)=0.68, d\left(C_{15}, A^{-}\right)=1.156\right.$

and $d\left(C_{16}, A^{-}\right)=0.23$

$\therefore D_{11}^{-}=0.6612+0.3983+0.5266+0.68+1.156+0.23=3.654$

Step 5: The closeness coefficient of each criterion, $C C_{i}$

By using Eq. (7) and the value of distance defined in the previous step, the closeness coefficient $C C_{i}$, calculated as following:

$$
C C_{1}=\frac{3.654}{5.015+3.654}=0.421,
$$

Repeat the same procedure to calculate $C C_{i}$ for each alternative. In the next step shows how the new criteria $C_{n+1}$ involved in the evaluation of Z- FRBS TOPSIS.

TABLE 5: IMPORTANCE OF CRITERIA
\begin{tabular}{|c|c|c|c|c|c|c|}
\hline & \multicolumn{2}{|c|}{ DM1 } & \multicolumn{2}{c|}{ DM2 } & \multicolumn{2}{c|}{ DM 3} \\
\cline { 2 - 8 } & $\boldsymbol{A}$ & $\boldsymbol{B}$ & $\boldsymbol{A}$ & $\boldsymbol{B}$ & $\boldsymbol{A}$ & $\boldsymbol{B}$ \\
\hline C1 & VH & L & VH & L & MH & SL \\
\hline C2 & MH & SL & MH & SL & MH & L \\
\hline C 3 & MH & SWL & M & SWL & H & SL \\
\hline C 4 & M & L & ML & L & M & L \\
\hline C 5 & H & SL & H & SL & VH & SWL \\
\hline C6 & ML & L & M & L & ML & L \\
\hline C7 & 8.00 & & 10.00 & & 7.00 & \\
\hline
\end{tabular}

TABLE 6(A): RATING BASED ON DM1 OPINION

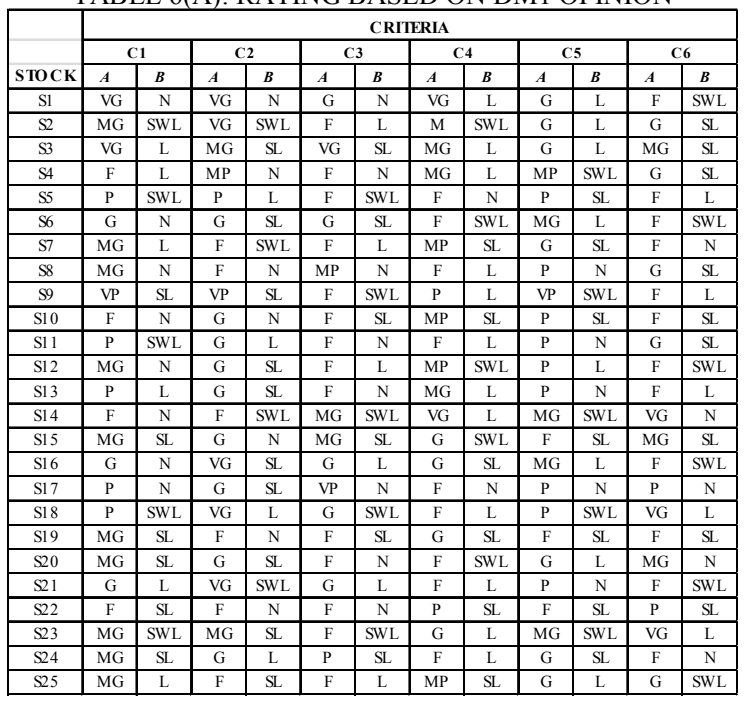

TABLE 6(B): RATING BASED ON DM2 OPINIONS

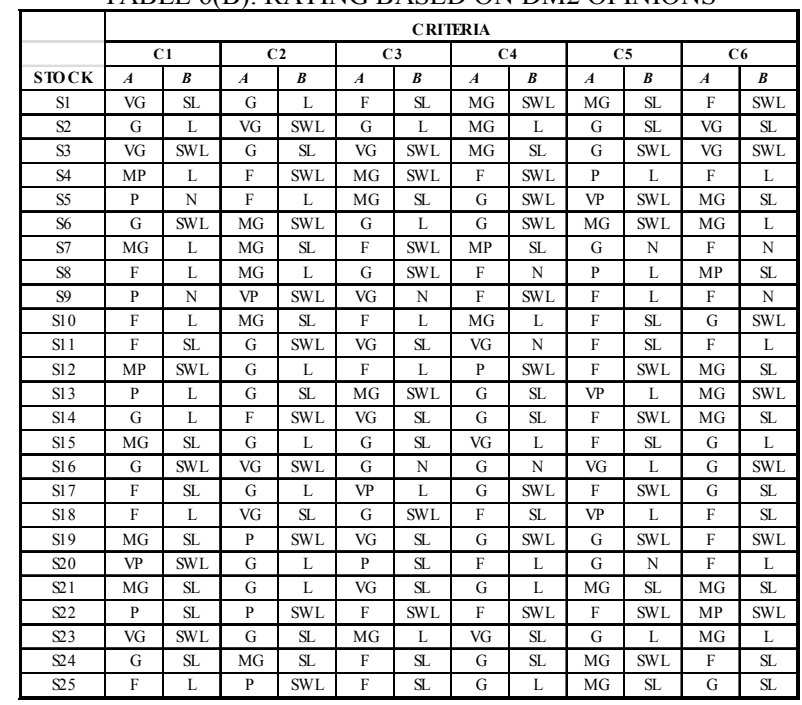

TABLE 6(C): RATING BASED ON DM3 OPINIONS

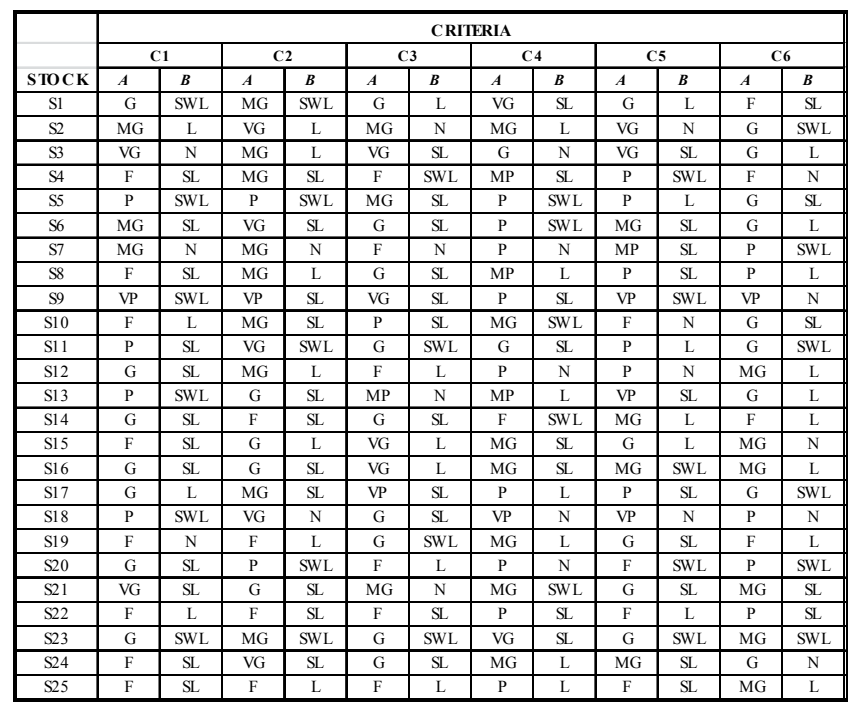

Step 6: The Influence Closeness Coefficient $\left(I C C_{i}\right)$ of each alternative

Firstly the influence degree $\left(\sigma_{K}\right)$ of each decision maker must be determined using Eq. (8) based on their experience on the domain. In this case study, $C_{7}$ in Table 5 represents the importance degree of decision maker. DMs evaluate themselves by giving value 0 to 10 , for unimportant and very importance respectively. For instance influence degree of DM1 is calculated as follows:

$$
\begin{gathered}
\sigma_{1}=\frac{8}{8+10+7} \\
=0.32
\end{gathered}
$$

Follow by Eq. (9) to get the influenced closeness coefficient for $A_{1}$

$$
\begin{aligned}
I C C_{1} & =0.32 \times 0.421 \\
& =0.135
\end{aligned}
$$


Finally, the influenced closeness coefficients need to be normalized before match the coefficient to the linguistic variable in Table 4. As an example in this case study, assuming the maximum value of ICC, $I C C_{i}=0.183$ then the normalized influenced closeness degree calculated as follows:

$$
\begin{gathered}
N I C C_{1}=\frac{0.135}{0.183} \\
=0.7386
\end{gathered}
$$

Step 7: The matrix of antecedent $(\Lambda)$ and the matrix of consequent $(\chi)$

Each decision maker has a matrix of antecedent and consequent separately.

$$
\text { If } \begin{gathered}
R_{1} \\
R_{2} \\
\vdots \\
R_{m}
\end{gathered}\left[\begin{array}{cccc}
X_{11} & X_{12} & \cdots & X_{1 n} \\
X_{21} & X_{22} & \cdots & X_{2 n} \\
\vdots & \vdots & \ddots & \vdots \\
X_{m 1} & X_{m 2} & \cdots & X_{m n}
\end{array}\right] \text { then }\left[\begin{array}{c}
Y_{1} \\
Y_{2} \\
\vdots \\
Y_{m}
\end{array}\right]
$$

The rules have the following format:

IF $X_{11}$ is VG and $X_{12}$ is $\mathrm{VG}$ and $X_{13}$ is $\mathrm{G}$ and $X_{14}$ is $\mathrm{VG}$ and

$$
X_{15} \text { is } \mathrm{G} \text { and } X_{16} \text { is } \mathrm{F} \text { Then the output } Y_{1} \text { is } \mathrm{G} \text {. }
$$

Now, the value of $N_{I C C}$ can be match to the linguistic variable for alternative in Table 4. For instance, $N I C C_{1}=0.7386$.Then value of $Y_{1}$ is belong to fuzzy set $\mathrm{G}$ in Table 4.

Step 8: The final score $(\Gamma)$ for each alternative.

Assuming S1 have three rules R1, R2 R3: For example final score for $\mathrm{S} 1$ is shown below:

$\mathrm{R} 1$ : if $\mathrm{C} 1$ is $\mathrm{VG}$ and $\mathrm{C} 2$ is $\mathrm{VG}$ and $\mathrm{C} 3$ is G AND C4 is $\mathrm{VG}$ and $\mathrm{C} 5$ is $\mathrm{G}$ and $\mathrm{C} 6$ is $\mathrm{F}$ Then $\mathrm{S} 1$ is $\mathrm{G}$

R2: if $\mathrm{C} 1$ is $\mathrm{VG}$ and $\mathrm{C} 2$ is $\mathrm{G}$ and $\mathrm{C} 3$ is $\mathrm{F}$ and $\mathrm{C} 4$ is $\mathrm{MG}$ and $\mathrm{C5}$ is MG and $\mathrm{C} 6$ is $\mathrm{F}$ Then $\mathrm{S} 1$ is $\mathrm{VG}$

R3: if $\mathrm{C} 1$ is $\mathrm{G}$ and $\mathrm{C} 2$ is $\mathrm{MG}$ and $\mathrm{C} 3$ is $\mathrm{G}$ and $\mathrm{C} 4$ is $\mathrm{VG}$ and $\mathrm{C} 5$ is $\mathrm{G}$ and $\mathrm{C} 6$ is $\mathrm{F}$ Then $\mathrm{S} 1$ is $\mathrm{VG}$

Let the output of each rule for $\mathrm{S} 1$ are

$\mathrm{R} 1: \mathrm{G}=(0.60,0.7,0.7,0.8)$

$\mathrm{R} 2: \mathrm{VG}=(0.80,0.9,0.9,1.0)$

$\mathrm{R} 3: \mathrm{VG}=(0.80,0.9,0.9,1.0)$.

Then, based on Eq. (14) the value of final score is calculated as follows

$$
\begin{gathered}
\lambda=\frac{0.7+0.9+0.9}{3} \\
=0.83
\end{gathered}
$$

From Step 6, by assuming ICC of each rule for S1 are

$$
\begin{aligned}
& \text { R1: } 0.7386 \\
& \text { R2: } 0.8990 \\
& \text { R3: } 0.9334
\end{aligned}
$$

The value of $\Omega$ defined as

$$
\begin{gathered}
\Omega=\frac{0.7386+0.8990+0.9334}{3} \\
=0.8569
\end{gathered}
$$

Lastly, the final score $\Gamma$ can be derived as Eq. (13)

$\Gamma=\lambda * \Omega$

$=0.83 \times 0.8569$

$=0.7141$

Therefore, from the value of $\Gamma$, the ranking order of all alternative is determined. The best alternative has higher value of $\Gamma$. The rank based on proposed method presented in Table 7.

\section{ANALYSIS OF RESULTS}

For the validation purposes, the authors consider the ranking based on existing non rule based approach and actual price change. The rankings are compared descriptively using Spearman rho correlation. The advantages of this correlation method are its easy algebraic structure and intuitively simple interpretation. Besides this, the method is less sensitive to bias due to the effect of outliers and can be used to reduce the weight of outliers (large distances get treated as a one-rank difference).

In general, the coefficient of rho $(\rho)$ measures the strength of association between two ranked variables. The formula used to calculate Spearman's Rank is shown below.

$$
\rho=1-\frac{6 \sum \partial_{i}^{2}}{n^{3}-n}
$$

where $\partial_{i}$ represents the difference between the ranks and $n$ donated number of alternatives considered. The Spearman correlation coefficient, $\rho$ can take values between +1 to -1 . If $\rho=1$ indicates a perfect relationship of ranks, if $\rho=0$ shows no relationship between ranks and $\rho=-1$ indicates a perfect negative association of ranks. The closer $\rho$ is to zero, the weaker the relationship between the ranks. Thus based on the analysis of Spearman rho correlation in Table 7, it is observed that the novel method (NM) Z-FRBS TOPSIS outperform the existing non rule based approach.

\section{CONCLUSION}

In this paper, a novel version of TOPSIS method using Znumbers by extending the ability of fuzzy rule based approach in multi criteria decision making analysis is presented. The main novelty of this paper is the modification of TOPSIS method by fuzzy rule based approach using Z-numbers by considering expert experience and knowledge in decision analysis. The proposed method not only provides a useful way to handle vagueness and imperfect information in decision making practice in more flexible and intelligent manner but also presents expert knowledge more accurately. The ranking based on proposed method is validated comparatively using 
Spearman rho correlation coefficient. The result shows proposed method outperform the existing non rule based version of TOPSIS in terms of ranking performance.

\section{TABLE 7: TOPSIS RANKING PERFORMANCE BASED ON SPEARMAN RHO CORRELATION}

\begin{tabular}{|c|c|c|c|c|c|c|c|}
\hline & \multicolumn{3}{|c|}{ Ranking } & \multicolumn{2}{|c|}{ Z } & \multicolumn{2}{c|}{ Z-FRBS(NM) } \\
\hline STOC K & $\boldsymbol{A c t} \boldsymbol{c} \boldsymbol{a} \boldsymbol{l} \boldsymbol{l}$ & $\boldsymbol{Z}$ & $\boldsymbol{Z} \boldsymbol{F R \boldsymbol { B } \boldsymbol { S }}$ & $\partial_{i}$ & $\partial^{2}$ & $\partial_{i}$ & $\partial^{2}$ \\
\hline S1 & 2 & 7 & 5 & -5 & 25 & -3 & 9 \\
\hline S2 & 4 & 9 & 10 & -5 & 25 & -6 & 36 \\
\hline S3 & 1 & 1 & 1 & 0 & 0 & 0 & 0 \\
\hline S4 & 21 & 20 & 20 & 1 & 1 & 1 & 1 \\
\hline S5 & 19 & 24 & 24 & -5 & 25 & -5 & 25 \\
\hline S6 & 11 & 8 & 9 & 3 & 9 & 2 & 4 \\
\hline S7 & 17 & 17 & 16 & 0 & 0 & 1 & 1 \\
\hline S8 & 24 & 18 & 18 & 6 & 36 & 6 & 36 \\
\hline S9 & 23 & 25 & 25 & -2 & 4 & -2 & 4 \\
\hline S10 & 22 & 14 & 14 & 8 & 64 & 8 & 64 \\
\hline S1 1 & 8 & 13 & 13 & -5 & 25 & -5 & 25 \\
\hline S12 & 13 & 16 & 15 & -3 & 9 & -2 & 4 \\
\hline S13 & 25 & 22 & 22 & 3 & 9 & 3 & 9 \\
\hline S14 & 9 & 10 & 8 & -1 & 1 & 1 & 1 \\
\hline S15 & 3 & 3 & 3 & 0 & 0 & 0 & 0 \\
\hline S16 & 5 & 2 & 2 & 3 & 9 & 3 & 9 \\
\hline S17 & 18 & 21 & 21 & -3 & 9 & -3 & 9 \\
\hline S18 & 12 & 19 & 19 & -7 & 49 & -7 & 49 \\
\hline S19 & 15 & 11 & 11 & 4 & 16 & 4 & 16 \\
\hline S20 & 16 & 15 & 17 & 1 & 1 & -1 & 1 \\
\hline S21 & 7 & 4 & 4 & 3 & 9 & 3 & 9 \\
\hline S22 & 20 & 23 & 23 & -3 & 9 & -3 & 9 \\
\hline S23 & 6 & 5 & 7 & 1 & 1 & -1 & 1 \\
\hline S24 & 14 & 6 & 6 & 8 & 64 & 8 & 64 \\
\hline S25 & 10 & 12 & 12 & -2 & 4 & -2 & 4 \\
\hline & & & & 0 & 404 & 0 & 390 \\
\hline Spearman Rho Coeefficient & & 0.845 & & 0.85 \\
\hline
\end{tabular}

\section{REFERENCES}

[1] X. Sun and Y. Li, “An Intelligent Multi-Criteria Decision Support System for Systems Design," 10th AIAA Aviat. Technol. Integr. Oper. Conf., pp. 1-11, Sep. 2010.

[2] A. Kelemenis, K. Ergazakis, and D. Askounis, "Support managers' selection using an extension of fuzzy TOPSIS," Expert Syst. Appl., vol. 38, no. 3, pp. 2774-2782, Mar. 2011.

[3] A. Awasthi, S. S. Chauhan, and H. Omrani, "Application of fuzzy TOPSIS in evaluating sustainable transportation systems," Expert Syst. Appl., vol. 38, no. 10, pp. 12270-12280, Sep. 2011.
[4] H. Shidpour, M. Shahrokhi, and A. Bernard, "A multi-objective programming approach, integrated into the TOPSIS method, in order to optimize product design; in three-dimensional concurrent engineering," Comput. Ind. Eng., vol. 64, no. 4, pp. 875-885, Apr. 2013.

[5] Y. T. İç, "An experimental design approach using TOPSIS method for the selection of computer-integrated manufacturing technologies," Robot. Comput. Integr. Manuf., vol. 28, no. 2, pp. 245-256, Apr. 2012.

[6] A. Pires, N.-B. Chang, and G. Martinho, "An AHP-based fuzzy interval TOPSIS assessment for sustainable expansion of the solid waste management system in Setúbal Peninsula, Portugal," Resour. Conserv. Recycl., vol. 56, no. 1, pp. 7-21, Nov. 2011.

[7] M. Saremi, S. F. Mousavi, and A. Sanayei, "TQM consultant selection in SMEs with TOPSIS under fuzzy environment," Expert Syst. Appl., vol. 36, no. 2, pp. 2742-2749, Mar. 2009.

[8] M. Dağdeviren, S. Yavuz, and N. Kılınç, "Weapon selection using the AHP and TOPSIS methods under fuzzy environment," Expert Syst. Appl., vol. 36, no. 4, pp. 8143-8151, May 2009.

[9] D. Mohamad and R. M. Jamil, "A Preference Analysis Model for Selecting Tourist Destinations based on Motivational Factors: A Case Study in Kedah, Malaysia," Procedia - Soc. Behav. Sci., vol. 65, pp. 20-25, Dec. 2012.

[10] T. Özcan, N. Çelebi, and Ş. Esnaf, "Comparative analysis of multicriteria decision making methodologies and implementation of a warehouse location selection problem," Expert Syst. Appl., vol. 38, no. 8, pp. 9773-9779, Aug. 2011.

[11] G. Kim, C. S. Park, and K. P. Yoon, "Identifying investment opportunities for advanced manufacturing systems with comparativeintegrated performance measurement," Int. J. Prod. Econ., vol. 50, no. 1, pp. 23-33, May 1997.

[12] H.-S. Shih, H.-J. Shyur, and E. S. Lee, "An extension of TOPSIS for group decision making," Math. Comput. Model., vol. 45, no. 7-8, pp. 801-813, Apr. 2007.

[13] C.-T. Chen, "Extensions of the TOPSIS for group decision-making under fuzzy environment," Fuzzy Sets Syst., vol. 114, no. 1, pp. 1-9, Aug. 2000.

[14] S.-M. Chen and L.-W. Lee, "Fuzzy multiple attributes group decisionmaking based on the interval type-2 TOPSIS method," Expert Syst. Appl., vol. 37, no. 4, pp. 2790-2798, Apr. 2010.

[15] Alireza Sotoudeh-Anvari and Soheil Sadi-Nezhad, "A new approach based on the level of reliability of information to determine the relative weights of criteria in fuzzy TOPSIS," Int. J. Appl. Decis. Sci., 2015.

[16] L. A. Zadeh, "A Note on Z-numbers," Inf. Sci. (Ny)., vol. 181, no. 14, pp. 2923-2932, Jul. 2011.

[17] F. J. J. Santos and H. A. Camargo, "Decision support systems in multicriteria groups: An approach based on fuzzy rules," Int. Conf. Fuzzy Syst., pp. 1-8, Jul. 2010.

[18] B. Kang, D. Wei, Y. Li, and Y. Deng, "A Method of Converting Znumber to," J. Inf. Comput. Sci., vol. 3, pp. 703-709, March 2012. 